\title{
Impregnability and performance of rCF-nonwovens with epoxy resin
}

\author{
Jasmin Mankiewicz ${ }^{1)}, E$ Enst Cleve ${ }^{1)}$, Michael Heber ${ }^{1)}$ and Jochen S. Gutmann ${ }^{2)}$ \\ 1) Institute for Coatings and Surface Chemistry, jasmin.mankiewicz@hs-niederrhein.de, University of \\ Applied Sciences, Adlerstraße 32, 47798 Krefeld, Germany \\ 2) Faculty of Chemistry, jochen.gutmann@uni-due.de, University Duisburg-Essen, Universitätsstraße \\ 2, 45141 Essen, Germany
}

\section{Keywords}

Lightweight, material properties, resin transfer molding, recycling, short carbon fibers, wet-laid

\begin{abstract}
Currently, the carbon fiber key market increases intensely. In consequence of production, $10-30 \%$ of cost-intensive carbon fiber waste is accumulated by blending fabrics, prepregs which are out of specification and end-of-life products. Because of a landfill ban for carbon fibers, environmental aspects and a cost reduction potential, there is a stronger focus on carbon fiber recycling [1]. Through new recycling methods, the carbon fiber is regained from polymer matrix, but looses its woven structure.
\end{abstract}

One possibility to re-use chopped recycled fibers is through a fiber mat. Carbon fiber nonwovens can be fabricated by a wet-laid process, for example. For recycled fibers without a specific fiber length and sizing, a challenge lies in separating them in dispersion to get homogenous nonwovens and interlink the fibers in the nonwoven. For the first step, stirring and surfactants improve the separation [2], hydroxyethylcellulose ensures the bonding. Then the nonwoven can be impregnated with thermosets by resin transfer molding (RTM). The University of Applied Science Niederrhein is the first to investigate the whole process chain from handling recycled carbon fibers in order to attain the finished composite. Optical impregnability is inspected and material properties (tensile strength and Young's modulus) are analyzed and compared to virgin fibers to get information on performance. A density range from $1.18(5 \% \mathrm{CF})$ to $1.4 \mathrm{~g} / \mathrm{cm}^{3}(40 \% \mathrm{CF})$ is very attractive for lightweight constructions as well.

\section{Introduction}

The increase in the carbon fiber market is caused by developments in the automotive sector, where electric powered mobility and decrease in weight play a major role. On the one hand, a bigger extent of electrically operated automobiles needs to be produced and sold. The best solution to compensate battery weight is using reinforced plastics (particularly carbon fiber-reinforced plastics). On the other hand, there are stringent EU requirements referring to conservation and $\mathrm{CO}_{2}$ reduction. Principally, emissions of combustion engines should be lowered from 141.8 grams $\mathrm{CO}_{2}$ per kilometer (2012) to 95 grams per kilometer until 2020 [3]. Therefore, basic materials have to be substituted under maintenance of physical and mechanical properties. This is possible with carbon fibers [4]. The composites market report 2016 forecasts a need of $120.0 \mathrm{kt}$ CFRP until 2022. This is a doubling of the current demand [3]. In consequence of production, 10-30\% of cost-intensive carbon fiber waste is accumulated by blending fabrics, prepregs which are out of specification and end-of-life products. Because of a landfill ban for carbon fibers, environmental aspects and a cost reduction potential there is a stronger focus on carbon fiber recycling [2]. Through new recycling methods the carbon fiber is regained from the polymer matrix. The fibers have properties very similar to virgin fibers, but loose their woven structure. There are three main types: material (molding mass), thermal (pyrolysis) and 
chemical recycling (solvolysis). Recent research deals with supercritical solvolysis of water and other fluids $[5,6]$.

One possibility to re-use chopped recycled fibers is through a fiber mat. Carbon fiber nonwovens can be fabricated by aerodynamics, hydrodynamics (wet-laid) or carding. The University of Applied Sciences Niederrhein uses the wet-laid process. Its advantages are an isotropic behavior of randomly oriented fibers, low costs in production, no formation of dust or respirable particulates, therefore no electrostatic and health problems when using this process and a low surface weight with maximum of homogeneity [7]. For recycled fibers without a specific fiber length and sizing, a special challenge lies in separating them in dispersion to get homogenous nonwovens and interlink the fibers in the nonwoven. Afterwards, the nonwoven can be impregnated with thermosets by resin transfer molding (RTM) and be reasonably used as a resource for automotive parts as roof lining, roof rack, and underbody, battery lock up, bike parts, sports goods or in the medical field for trays.

\section{Materials}

\subsection{Dispersing Agent and Surfactant}

For each dispersion, a stock solution with hydroxyethyl cellulose from SE Tylose GmbH \& Co. KG and demineralized water is prepared. Subsequently $587.5 \mathrm{~g}$ stock solution $(80 \mathrm{~g} / \mathrm{L})$ is weighted and filled up to $2,000 \mathrm{~g}$ by demineralized water. To improve the separation of recycled fibers $0.5 \%$ to $4 \%$ surfactant Tego STO 85 V from Evonik Nutrition \& Care GmbH was added to the dispersion.

\subsection{Carbon Fibers}

Recycled carbon fibers (rCF) from ELG Carbon Fibre Ltd., recovered by pyrolysis, with a fiber length of 30 to $60 \mathrm{~mm}$ without sizing (further on rCF1) and from Altex Textil-Recycling $\mathrm{GmbH} \& \mathrm{Co}$. KG (delivered by Johann Borgers $\mathrm{GmbH}$ ) with an average fiber length of $41 \mathrm{~mm}$ (further on rCF2) were used. Both are cut to a length of $12 \mathrm{~mm}$ for the comparison and then nonwovens with $1 \mathrm{~g}$ carbon fiber content are laid down with reduced length and original length.

\subsection{Resin system}

With a low viscosity bisphenol-A resin (EPIKOTE ${ }^{\mathrm{TM}}$ resin MGS RIMR426) and an amine-based hardener (EPICURE Curing Agent RIMH 433) delivered by Lange+Ritter GmbH, a reaction time of 2 hours can be realized at room temperature. The resin hardener ratio is continual 100 to 26 . The mechanical properties of the data sheet are $65 \mathrm{MPa}$ tensile strength and $2700 \mathrm{MPa}$ E-Modulus.

\section{Methods}

\subsection{Wet laid process}

For separation of the fiber bundles, $1 \mathrm{~g}$ is added to the dispersion with $3 \%$ of surfactant and stirred for 30 minutes. Subsequently, the dispersion is filled into a sheet former (see in Figure 1) and a nonwoven is formed by the Rapid-Köthen method. When the cylinder is filled to seven liters, air is swirled from below through the dispersion (20 seconds). Afterwards, the mixture is deoxidized (15 seconds) and the cylinder is dewatered. As a result, the fibers are deposited on the net as a nonwoven with lower density. A nonwoven has a diameter of $200 \mathrm{~mm}$. Finally, when the nonwoven is dried, it can be removed from the net. 

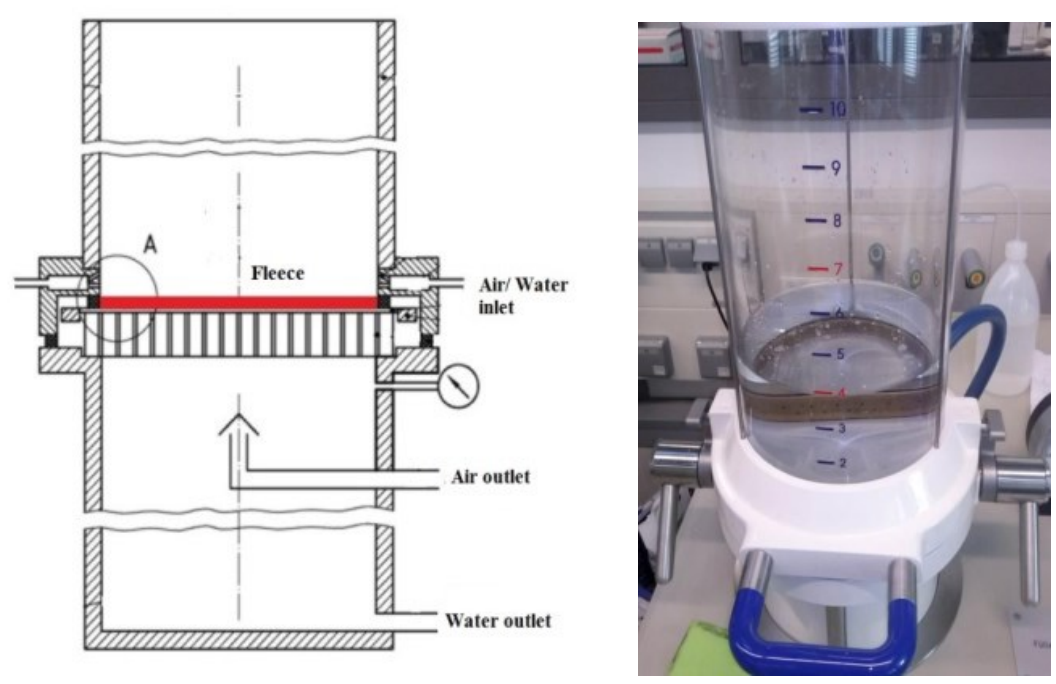

Figure 1: Schematic (left side) and real (right side) setup for the

Rapid-Köthen method according to DIN EN ISO 5269-2 [9]

\subsection{Resin-Transfer-Molding (RTM)}

Composites are manufactured with the nonwovens and the mentioned epoxy resin system by RTM. For this purpose, the injection unit iJect touch 2.0 (see Figure 2, left side) from Wolfangel $\mathrm{GmbH}$ is utilized. This machine keeps different parameters constant, such as injection pressure, material temperatures and quantities. Increasing pressure during injection causes complete impregnation and replacement of air in the composite. Accurate quantities avoid unnecessary waste getting out of the venting nozzle.
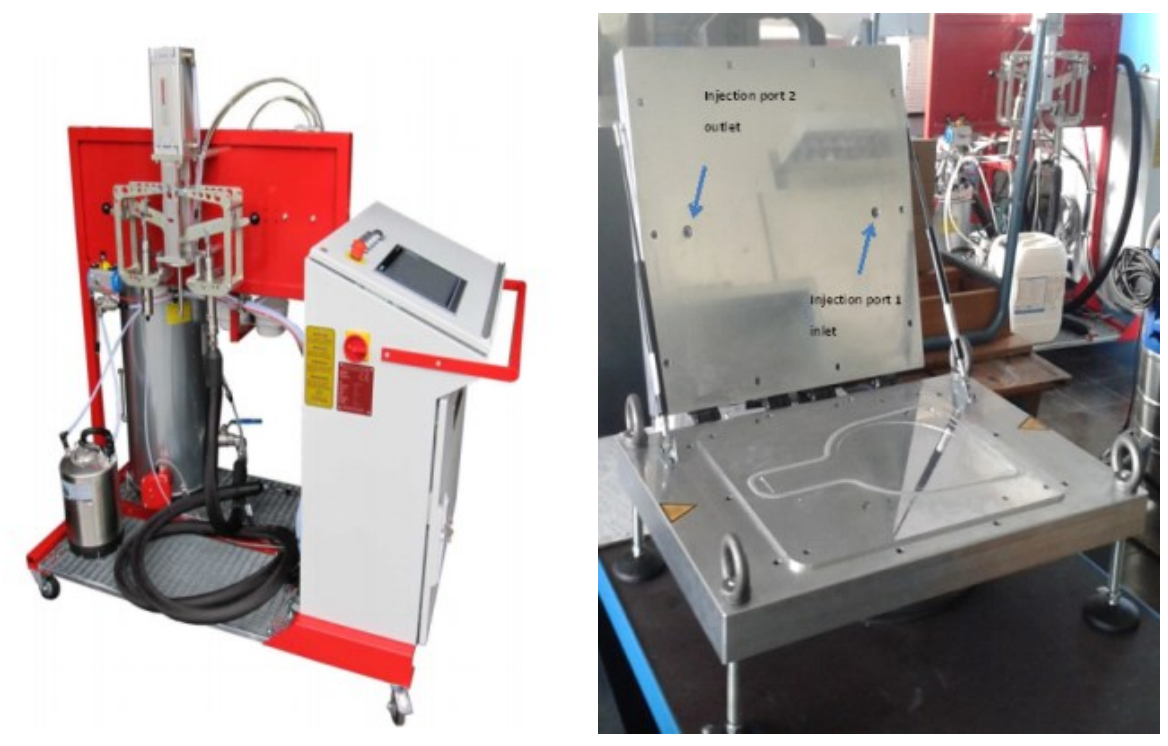

Figure 2: Injection unit (left side) and aluminum tool (right side).

The injection unit is connected with the injection nozzle (on top of the aluminum tool) by pipe (Figure 2, right side). The tool has a round cavity with heating panels on top and bottom to control the reaction rate. For first results, only three to six nonwovens are stacked in the cavity. After insertion, the tool is closed and the mold cavity is filled with the resin under pressure ( 0.5 bar up to a maximum of 3 bar). The chemical reaction takes place within two hours. When the disk is hardened, it can be removed from the mold. The disks have a final thickness of $1 \mathrm{~mm}$ and fiber volume content of $5 \%$ or $10 \%$. To get the final properties the disc has to be post-cured for 10 hours at $80^{\circ} \mathrm{C}$. Optical impregnability is inspected and can be compared to composites consisting of virgin fibers. 


\section{Results}

\subsection{Nonwoven Formation of rCF}

For the fiber separation, a concentration of $3 \%$ surfactant showed the best results. At lower concentrations some fiber bundles could still be observed, even though with $2 \%$ surfactant the separation of fibers could be clearly seen. On the other hand, higher concentrations promote the winding of fibers around the stirrer. These results are shown in Figure 3.
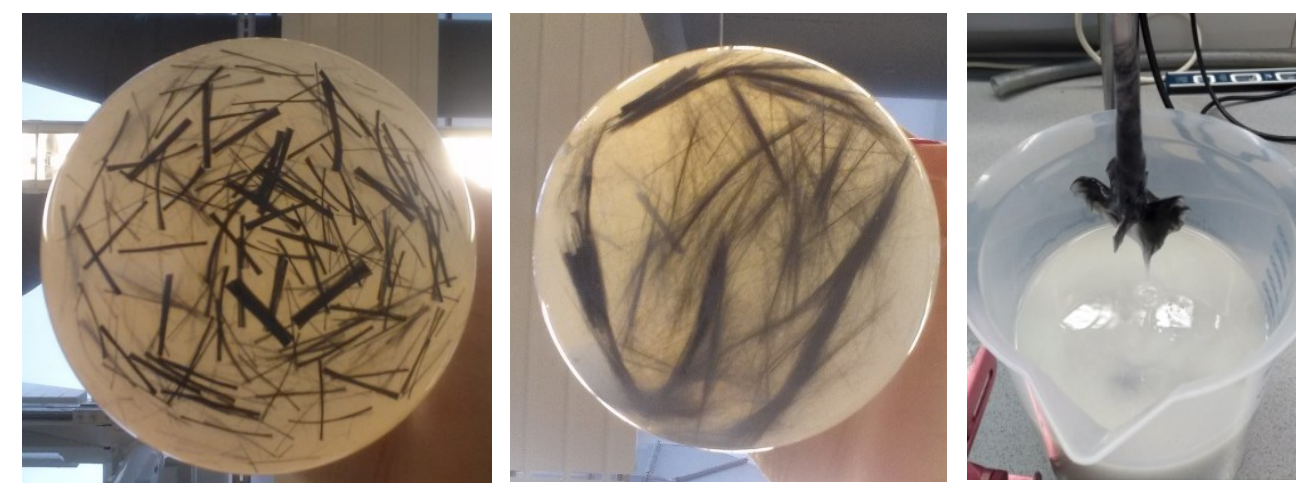

Figure 3: Behavior of rCF with $0.5 \%$ surfactant (left side), $2.5 \%$ (middle) and $>3 \%$ surfactant (right side)

With addition of the surfactant, the water became opaque and the diversification of the fibers during the stirring process was hard to see. This is because an emulsion is created. Figure 4 depicts the difference between the dispersion, dispersion with recently added rCF1 and after a stirring time of 20 minutes. In conclusion, a stirring time of 20 to 30 minutes is the optimum. Afterwards, fibers start to wind around the stirrer and become agglutinations in the dispersion as well as on the nonwoven later on. This decreases the material properties as found in prior studies.
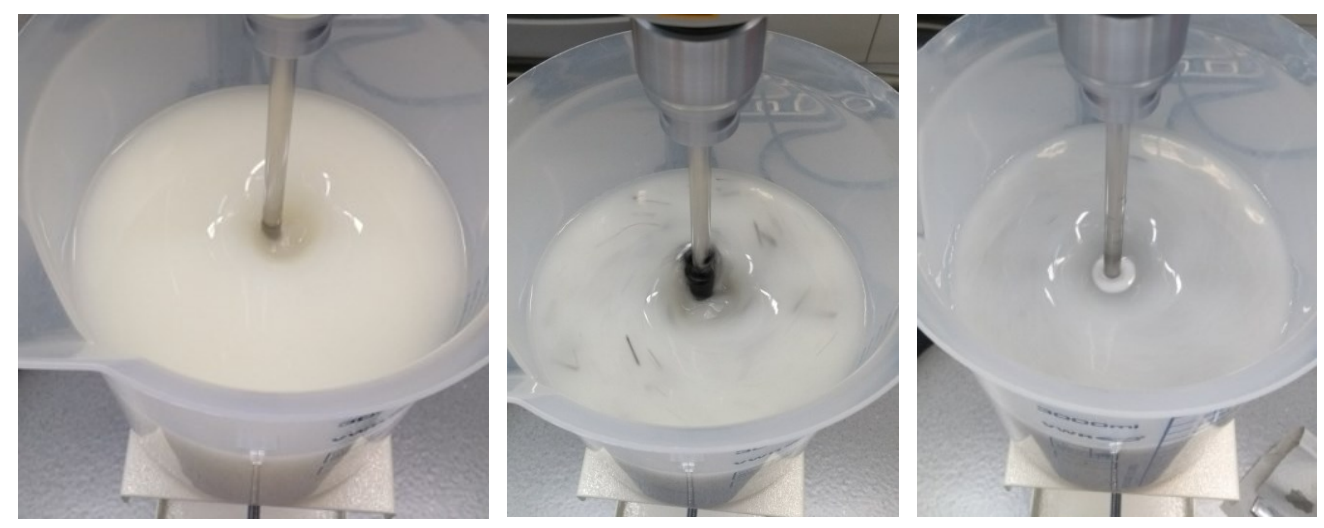

Figure 4: Behavior of the dispersion without and with fibers added and different stirring times

After stock preparation the whole dispersion was poured in the sheet formation plant and treated as mentioned before. The obtained nonwoven is optically similar to the virgin fiber nonwoven but the surface feel is softer, as shown in Figure 5. 

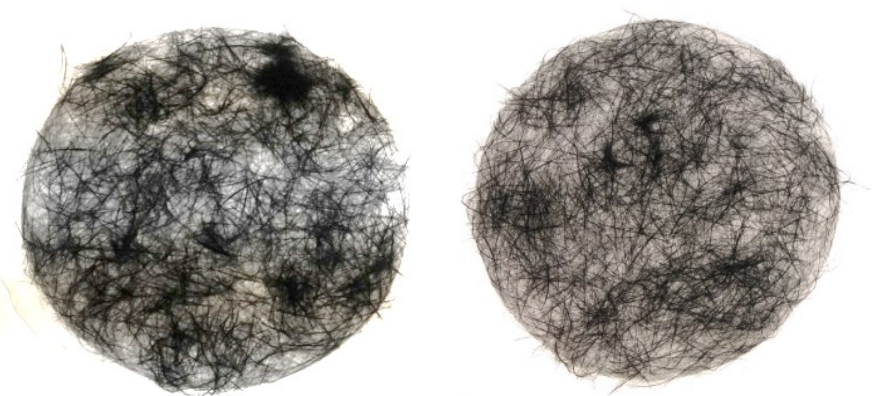

Figure 5: Comparison of virgin fiber nonwoven (left side) and rCF1 nonwoven (right side)

For the rCF2, the stirring was more difficult. These fibers agglomerate more easily, so that they were stirred for a shorter time, then filled directly into the plant and separated by the gassing step.

\subsection{Impregnability of rCF nonwoven}

The volume fractions of $5 \%$ and $10 \%$ are impregnated by a four-step pressure program in the RTM similar to the program that is used for virgin fibers. In the First quarter 0.3 bar are used to fill the empty cavity parts and ensure less air in the resin front. After that, the pressure is gradually increased every two strokes for 0.5 bar up to 2 bar and there is a holding pressure of 3 bar for 5 minutes after injection. There are no defects on the plate, the nonwovens are completely impregnated and have a smooth surface.
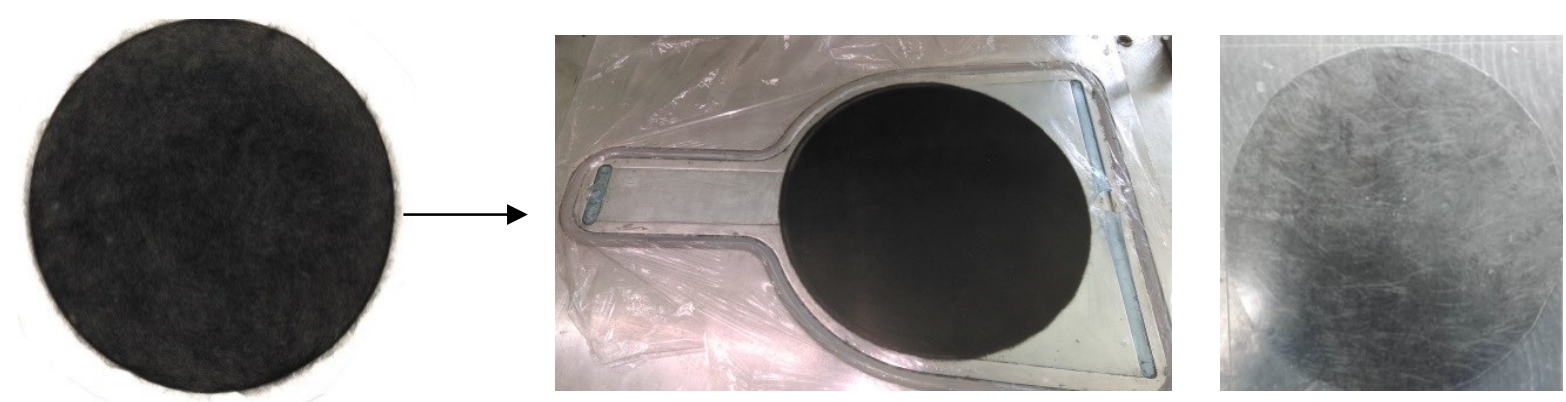

Figure 6: Single carbon fiber nonwoven (left side), virgin carbon fiber-epoxy disc in the cavity ( $d=200 \mathrm{~mm}$, middle) and a rCF nonwoven (right side)

The nonwoven with recycled fibers show no weakness compared to virgin fiber nonwoven with the same fiber volume friction and fiber length. Therefore, it is proven that recycled fibers conserve their performance over the whole process chain and show the same fiber matrix bonding as virgin fibers in the composite. Compared to the resin system itself, an improvement of $26 \%$ for the tensile strength and of $18 \%$ for the E-modulus is achieved. The strain is reduced to $50 \%$ and has an absolute value of $3.3 \%$ which is typical for this kind of reinforcement (see Figure 7 ).
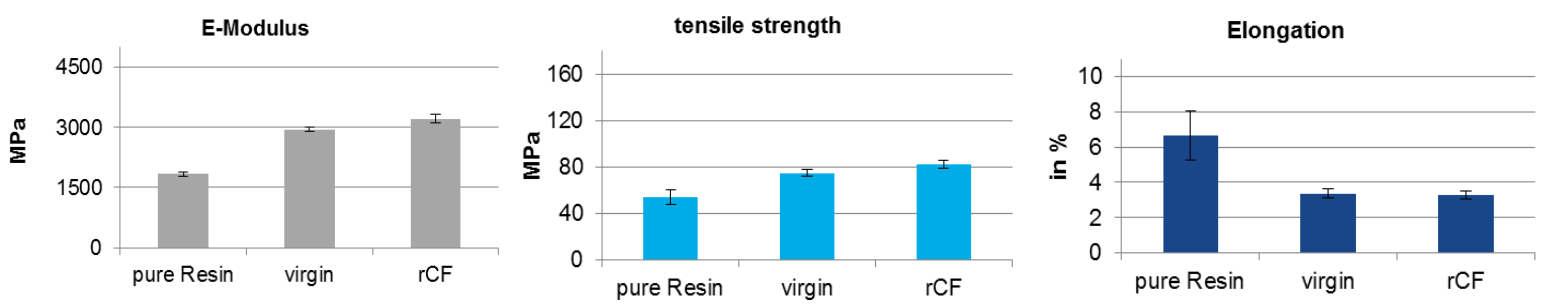

Figure 7: Comparison of mechanical properties of pure resin, virgin and recycled fibers (example $5 \%$ rCF1 in composite) 
With higher fiber volume fractions the properties can be increased nearly linearly. Thus, high fractions of recycled carbon fibers can be used for mid-tech lightweight parts.

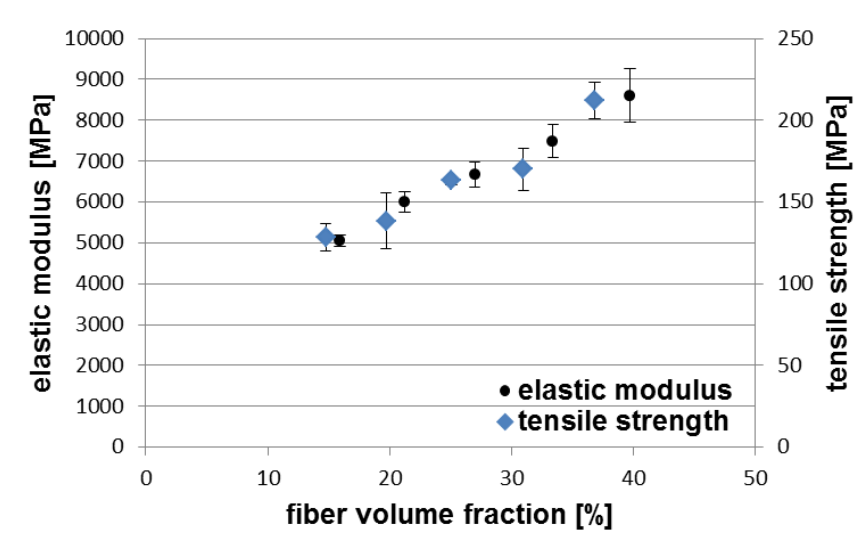

Figure 8: Course of material properties of CF nonwoven composite depending on fiber volume fraction

\section{Discussion and conclusion}

In this study, rCF were successfully dispersed and wet-laid nonwovens could be produced on a laboratory scale. Two different $\mathrm{rCF}$ were used and recycled fibers could be fully impregnated with an epoxy resin system. Compared to the virgin fiber samples, they show the same behavior during the manufacturing process and are similar concerning their mechanical results. Therefore, it is proven that pyrolysis fibers, whose individual properties are similar to virgin fibers, can conserve this performance over the whole process chain and show the same fiber matrix bonding as virgin fibers in the composite. The whole process chain could also be represented by this research and first information for a rCF nonwoven composite can be recorded in a data sheet. Thus, further investigations can be conducted more easily. Nonwovens will be produced on an industrial scale to show that they are suitable for series production and therefore a commercial re-use of recycled carbon fibers is possible, for example as battery lock ups or covers for batteries of e-bikes.

\section{Acknowledgments}

The project belonging to this paper is supported by the German Ministry of Education and Research with the funding code 03FH042PX4. Responsible for the papers content is its author.

\section{References}

[1] Hofmann, M.; Gulich, B.: Non-woven fabrics for the composite manufacturing - the recycling concept for carbon waste in long fiber form. Presentation, 27. Hofer Vliesstofftage, 2012.

[2] Vaisman, L.; Wagner, H. D.; Marom, G.: The role of surfactants in dispersion of carbon nanotubes. Adv. in Colloid and Interface Sci., 128-130 (2006), pp. 37-46. doi: 10.1016/j.cis.2006.11.007

[3] Kraus, T.; Kühnel, M.: Composites market report 2016 - market developments, trends, outlook and challenges. Düsseldorf, 2016. https://www.carbon-composites.eu/media/2449/market_report_2016_ccev-avk.pdf

[4] Verkehrsclub Deutschland: EU sets standards for passenger cars. 18 December 2016. https://www.vcd.org/themen/auto-umwelt/co2-grenzwert/

[5] Morin, C.; Loppinet-Serani, A.; Cansell, F.; Aymonier, C.: Near- and super-critical solvolysis of carbon fibre reinforced polymers (CFRPs) for recycling carbon fibers as a valuable resource: State of the art. J. of Supercrit. Fluids, 66 (2012), pp. 232-240. doi: 10.1016/j.supflu.2012.02.001 
[6] Schneller, A.; Henry, L.; Dörfler, J.; Müller, W.-M.; Aymonier, C.; Horn, S.: Recycling of carbon fibers from carbon fiber reinforced thermoset polymers by us of sub- and supercritical fluids. Presentation, ECCM17 - 17th European Conference on Composite Materials, Munich, 2016.

[7] Lützkendorf, R; Knobelsdorf, C.: Chances and possibilities of new products based on wet-laid process. Presentation, Thuringian Institute for Textile and Plastics Research, Rudolstadt, 2009.

[8] DIN EN ISO 5269-2: Pulps Preparation of laboratory sheets for physical testing - Part 2: Rapid-Köthen method, Berlin, 2005. 